\title{
The Standard Instrumentation Feedthrough System for the LHC Cryo-Magnets
}

\author{
D. Bozzini
}

\begin{abstract}
For the LHC, 1232 dipole magnets and about 400 quadrupole magnets operating at $1.9 \mathrm{~K}$ are installed in arcs and dispersion suppressors. Each cryo-magnet assembly comprises the main magnet and several small corrector magnets. Each assembly is equipped with voltage taps, quench heaters and cryogenic instrumentation. The number of instrumentation wires serving each magnet assembly and passing from cold to ambient is between 36 and 40.

An Instrumentation Feedthrough System (IFS) will electrically and mechanically connect the instrument wires to the outside of the vacuum vessel. The IFS has to satisfy several requirements: simplicity of integration, optimal access during tests and commissioning, voltage withstand and reliability during the lifetime of the machine. The heat load to superfluid helium must be minimized, and the long-term stability of the insulation vacuum should be preserved.

The solution foresees an open stainless steel tube housing the wires connected between the magnet and the outside of the vacuum vessel and terminated by a leak tight connector. The IFS is assembled from different standard components designed to fulfill the electrical, mechanical and thermal requirements and will be integrated by the industries that build the magnets. The standardization of the IFS for all types of cryo-magnet assemblies permits the same hardware interfaces and procedures to be used from the integration to commissioning and operation of the machine.

This paper describes the main parameters, the technical choices, the performance and the integration techniques of the IFS system.
\end{abstract}

\section{INTRODUCTION}

$\mathbf{T}$ HE INSTRUMENTS which are installed in the cryo-magnets of the LHC for diagnostic and protection require between 36 to 40 wires to be routed from the $1.9 \mathrm{~K}$ environment to the outside. The wires are put into a single stainless steel tube welded to the cold mass at one end, and to the outer shell of the cryostat at the other end. This type of Instrumentation Feedthrough System (IFS) will be used for most of the superconducting magnets in the LHC. The design is the result of theoretical studies and laboratory tests. Prototypes were installed in dipole magnets and Short Straight Sections (assembly of a quadrupole and several corrector magnets, together with components for cryogenics, vacuum and beam monitoring). The magnets for String 2 [1], composed of two LHC half-cell, are all equipped with the standard IFS adapted for prototype requirements.

Section II of this paper resumes the main parameter of the IFS based on the general parameters of the LHC machine and Section III discusses some of the technical choices. Details of the design are reported in Section IV and the integration technique is presented in Section V.

Manuscript received September 24, 2001.

Publisher Item Identifier S 1051-8223(02)04176-3.
TABLE I

MAIN PARAMETERS OF THE STANDARD IFS

\begin{tabular}{|c|c|c|}
\hline Parameter & Limit & Unit \\
\hline Radiation dose $e^{(1)}$ & 14000 & Gy \\
\hline Operating pressure & $1.310^{5}$ & $\mathrm{~Pa}$ \\
\hline Maximum pressure following a quench & $2010^{5}$ & $\mathrm{~Pa}$ \\
\hline Maximum rate of pressure rise during a quench & $10^{7}$ & $\mathrm{Pas}^{-1}$ \\
\hline Estimated number of pressure rises (quenches) & 9 & {$[-]$} \\
\hline Test pressure & $2510^{5}$ & $\mathrm{~Pa}$ \\
\hline Helium to atmosphere leak tightness ${ }^{(2)}$ & $<10^{-3}$ & Pa.m ${ }^{3} \mathrm{~s}^{-1}$ \\
\hline Ins. vacuum to atmosphere leak tightness ${ }^{(2)}$ & $<10^{-9}$ & Pa.m $\mathrm{s}^{3} \mathrm{~s}^{-1}$ \\
\hline Helium to ins. vacuum leak tightness ${ }^{(2)}$ & $<10^{-11}$ & Pa. $\mathrm{m}^{3} \mathrm{~s}^{-1}$ \\
\hline Thermal cycles $(300-1.9-300[\mathrm{~K}])^{(3)}$ & 25 & {$[-]$} \\
\hline Heat loads at $1.9 \mathrm{~K}$ & $<500$ & $\mathrm{~mW}$ \\
\hline Voltage withstand at warm ${ }^{(4)}$ & 5 & KV \\
\hline Voltage withstand at cryogenic condition ${ }^{(4)}$ & 3.1 & $\mathrm{KV}$ \\
\hline
\end{tabular}

\section{MAIN PARAMETERS}

As a sub assembly of a cryo-magnet, the standard IFS shall satisfy the main parameters listed in Table I. The large quantity of IFS to be installed and the industrial production and assembly impose some additional requirements. To minimize cost and to maximize reliability, commercially available, technically proven and reliable components are used. Simple and repeatable integration procedures shall also be used to guarantee a high reliability during installation and lifetime of the machine.

\section{TECHNICAL CHOICES}

Most instruments are for the protection of the magnet (voltage taps, powering of quench heaters). Four wires are dedicated to the precise measurement of the liquid helium temperature during normal operation and some wires to heaters for the acceleration of the warm-up phase. For simplicity, all instrumentation wires are introduced in the same IFS tube. Possible cross-talk between signals are acceptable since this is only expected during fast transients, for example a quench.

The IFS has been designed such that inter-connections of wires at cold are not required. Incidents occurring on prototype magnet show that cold electrical connections are delicate. The repair of such connections is difficult and would take a long time. In the LHC dipole magnets, an instrumentation wire is soldered to the sensor at cold, and to a pin of the room temperature feedthrough [2]. The connection placed at room temperature on the top of the vacuum vessel could be easily accessed in case of failure. The absence of cold connections will have a positive impact on the IFS system reliability, on cabling errors, assembly time and cost. An electrical connection should 


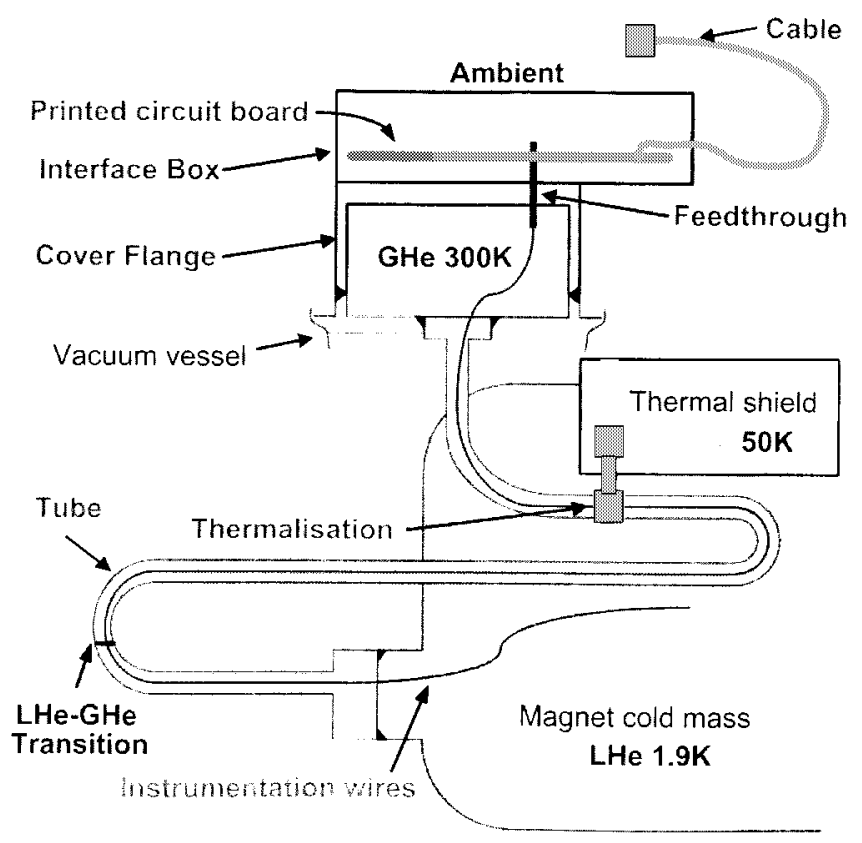

Fig. 1. The standard IFS.

be considered only in the case that the IFS would have to be changed. A polyimide insulation was chosen for the instrumentation wires in helium environment, to ensure that the voltage withstand requirements are fulfilled during the whole lifetime of the machine in spite of the high radiation dose [3].

The instrumentation wires will be connected to electronics placed under the cryo-magnet at a maximum distance of 14 meters. The cables joining the IFS to the electrical racks are installed during IFS assembly, and no additional cables and connectors are required between the IFS and the electronics. This solution allows testing the complete path of the instrumentation wires from the source of the instrument inside the cold mass to the electronics. Before the installation in the machine the entire system will be actively tested during the cryogenic tests of the magnets. Then the magnets will be installed in the tunnel, and it is sufficient to plug the cables with the connectors into the electronics.

\section{DESIGN OF THE STANDARD IFS}

The functional design of the standard IFS is shown in Fig. 1. The cold head of the IFS tube containing the wires is welded at the extremity of the cold mass.

The warm head of the IFS is welded to the vacuum vessel. The two welds guarantee the leak tightness between cold mass and insulation vacuum. The wires are connected at warm to the feedthroughs supported by a cover flange. The cover flange is welded to the vacuum vessel. The weld of the cover flange to the vacuum guarantees the tightness between the outside of the cryostat and the cold mass. The pressure withstand of the whole IFS is verified during the global pressure tests of the cold mass. At ambient, the wire feedthrough pins are soldered to a printed circuit board installed into a metallic interface box. For all instruments required for normal operation, cables connected to the printed circuit board bring the wires directly to the electronics. Wires used only for the diagnostic and commissioning of the

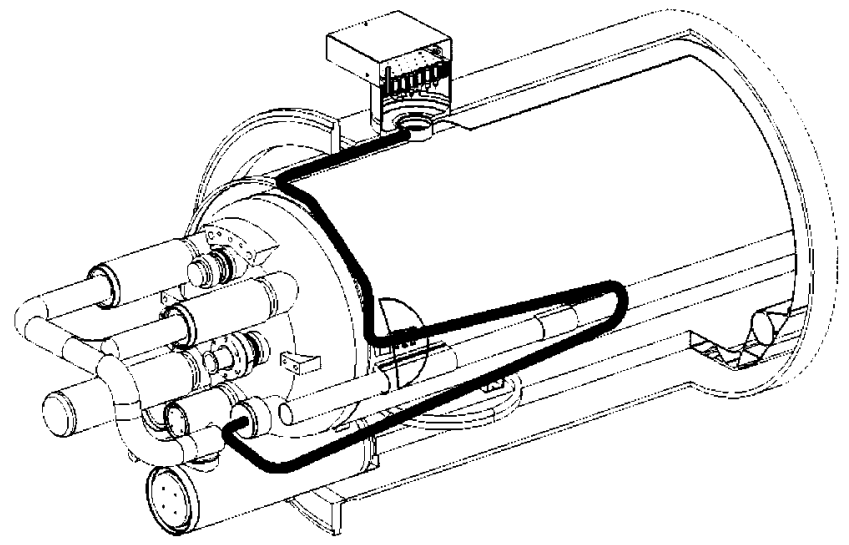

Fig. 2. Assembly view of the cryo-dipole IFS (cables are not shown).

machine will be connected from the printed circuit board to connectors at the interface box. During normal operation, the temperature varies along the IFS tube from $1.9 \mathrm{~K}$ in the cold mass to $300 \mathrm{~K}$ at the vacuum vessel flange. To limit the heat load at the $1.9 \mathrm{~K}$ level, the IFS tube is thermalized to a thermal shield operating around $50 \mathrm{~K}$. No vacuum or cryogenic seal or joint is used. An assembly view of the cryo-dipole IFS is shown in Fig. 2.

\section{A. Thermal Performance}

Heat loads to the superfluid helium bath are introduced via the IFS tube and the instrumentation wires. The filling ratio of the tube will define the level at which the liquid to gas transition will appear. The optimal situation is to maximize the length of the tube and aim at a filling ratio of the order of $70 \%$. A mathematical model has been developed to optimize the length and the filling ratio of the IFS tube in order to have the optimal thermal performance but also considering the mechanical integration of the wires into the IFS tube. To further reduce the heat load, mechanical thermalization as discussed above and multi-layer insulation blankets have been considered for the final design.

The thermal performances of different configurations of the Standard IFS have been tested in the laboratory [4]. It turned out that the most efficient way to decrease heat loads to the $1.9 \mathrm{~K}$ temperature is by thermalizing via the thermal screen. Finally, the retained parameters for the tube are a length of $3 \mathrm{~m}$ and an internal diameter of $8 \mathrm{~mm}$, with $1 \mathrm{~mm}$ wall thickness. The measured heat loads to $1.9 \mathrm{~K}$ amount to $300 \mathrm{~mW}$. Calculated heat loads to the screen via the thermalization are of the order of $500 \mathrm{~mW}$. Both values are within the allowed budget.

\section{B. Voltage Withstand}

During the manufacturing and operational life of each cryo-magnet the electrical withstand of the high voltage instrumentation will be tested at different voltage levels [5]. The worst voltage withstand condition will be the insulation to ground test at $3.1 \mathrm{kV}$, performed at cryogenic conditions.

Liquid helium at $1.9 \mathrm{~K}$ will be at the cold extremity of the IFS tube and warm gaseous helium at working pressure will be in the cover flange volume, where instrumentation wires are connected to the feedthroughs. The dielectric strength characteristic of the gaseous helium at $300 \mathrm{~K}$ [6] is approximately 6 


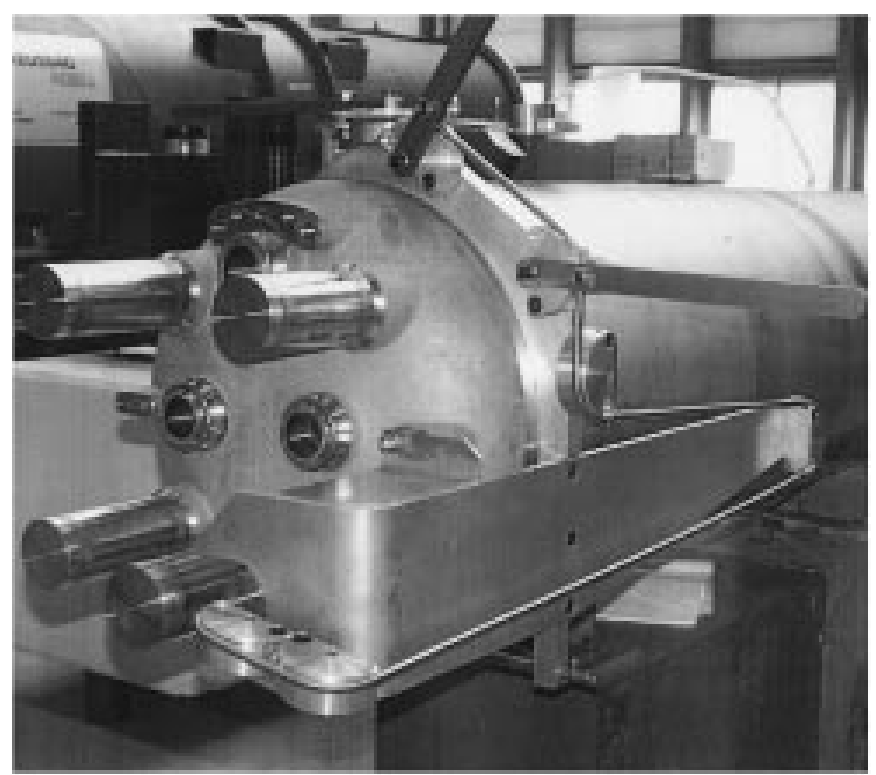

Fig. 3. Tooling for the IFS tube bending operation on a dipole magnet.

to 7 times worse than the dielectric strength of the air, therefore the feedthrough has been designed to withstand at least $21 \mathrm{kV}$ in air. Such a feedthrough is not available as an "off the shelf" component and has therefore been specially designed for this application. A minimum pin-to-pin or pin-to-ground distance of $25 \mathrm{~mm}$ is required to fulfill the worst electrical test.

\section{INTEGRATION TECHNIQUE}

The simplicity of the IFS minimizes the knowledge and technical competence required from the external contractors which will be charged with its integration. The IFS is assembled in two different phases. The first one, the assembly of the inner cryostat components, consists of introducing the wires into the tube and subsequently bending the tube. The second phase, the assembly of the outer cryostat components, consists of welding the tube to the vacuum vessel, then electrically connecting the wires to the feedthrough, welding the cover flange and finally installing the interface box.

The absence of cold electrical connections and the high filling ratio of the wires inside the tube impose to first introduce the bundle of wires into the straight tube and then bend the tube to its final shape. Fig. 3 shows the tooling foreseen for the bending on a dipole magnet IFS tube.

Integration steps are supplemented by ad hoc electrical verifications in order to detect problems related to the assembly as early as possible. A global electrical verification is required at the end of phase 1 and 2 . One single person can do the assembly of the complete IFS and the total time needed is of the order of 3 hours for phase 1 and 4 hours for phase 2, including the time for electrical verification.

\section{CONCLUSION}

Several prototypes of IFS have been built and tested. A number of technologies, components and sub-assemblies have been subjected to dedicated tests. All this has lead to the current design of the standard IFS.

Presently, five dipole magnets for the LHC machine have been equipped with the standard IFS and successfully tested at cryogenic conditions on the test bench.

\section{ACKNOWLEDGMENT}

M. Struik has participated in an early phase of this work. Thermal tests were performed at the Cryolab under the supervision of J.-M. Rieubland. Integration studies and drawings were prepared by T. Renaglia. Prototype construction, as well as dedicated mechanical, electrical and integration tests were carried out by A. Jacob and F. Gavin. Thanks to R. Schmid and G. Vandoni for critically revising the manuscript.

\section{REFERENCES}

[1] R. Saban et al., "The commissioning of the LHC test string 2,", Chicago, USA, LHC Project Report 480, Presented at the Particle Accelerator Conf. (PAC2001), June 18-22, 2001.

[2] G. Vandoni et al., "Instrumentation wires, connection techniques and feedthroughs for the main arc LHC cryomagnets and the QRL,", CERN, LHC-QY-ES-0001.00 rev.2.0.

[3] G. Brun, "Qualification of a material and a process for insulation the LHC instrumentation wiring," CERN, LHC Project Note 227, June 9, 2000.

[4] G. Vandoni et al., "The standard Instrumentation feedthrough system thermal performance," CERN, unpublished.

[5] F. Rodriguez-Mateos, "Voltage withstand levels for electrical insulation tests on components and busbar cross sections for the different LHC machine circuits,", CERN, LHC-PM-ES-0001.00 rev.1.0.

[6] K. H. Mess, P. Schmüser, and S. Wolff, Superconducting Accelerator Magnets. Singapore: World Scientific Publishing Co. Pte. Ltd., 1996, pp. 201-202. 